\title{
On the existence of a reverse shock in magnetized gamma-ray burst ejecta
}

\author{
D. Giannios ${ }^{1}$, P. Mimica ${ }^{2}$, and M. A. Aloy ${ }^{2}$ \\ 1 Max Planck Institute for Astrophysics, Box 1317, 85741 Garching, Germany \\ e-mail: giannios@mpa-garching.mpg.de \\ 2 Departamento de Astronomía y Astrofísica, Universidad de Valencia, 46100 Burjassot, Spain
}

Received 26 October 2007 / Accepted 23 November 2007

\section{ABSTRACT}

\begin{abstract}
The role of magnetic fields in gamma-ray burst (GRB) flows remains controversial. The study of the early afterglow phases and, in particular, of the reverse shock dynamics and associated emission offers a promising probe of the magnetization of the ejecta. In this paper, we derive the conditions for the existence of a reverse shock in arbitrarily magnetized ejecta that decelerate and interact with the circumburst medium. Both constant and wind-like density profiles are considered. We show, in contrast to previous estimates, that ejecta with magnetization $\sigma_{0} \gtrsim 1$ are not crossed by a reverse shock for a large fraction of the parameter space relevant to GRB flows. Allowing for shell spreading, there is always a relativistic or mildly relativistic reverse shock forming in $\sigma_{0} \lesssim 0.3$ ejecta. From this, we conclude that the paucity of optical flashes, believed to be a distinctive signature of a reverse shock, may be explained by the existence of dynamically important magnetic fields in the ejecta.
\end{abstract}

Key words. gamma rays: bursts - magnetohydrodynamics (MHD) - shock waves

\section{Introduction}

The role of magnetic fields in the GRB flow remains uncertain. Neutrino annihilation may be the dominant process that leads to the launching of the GRB flow by forming a fireball, i.e. a flow initially dominated by its thermal energy (Goodman 1986; Paczynski 1986). Alternatively, if they are powerful enough and develop the appropriate topology, magnetic fields can efficiently extract the rotational energy from the central engine launching a Poynting-flux-dominated flow (hereafter PDF; Usov 1992).

In the fireball model the magnetic fields are dynamically unimportant. The GRB flow during the onset of the afterglow phase is expected to be, at most, weakly magnetized. Though model dependent, the conversion of magnetic into kinetic energy in a PDF is partial (Michel 1969; Li et al. 1992; Vlahakis \& Königl 2003; Giannios \& Spruit 2006). The flow is expected to have a significant fraction of its energy in the form of Poynting flux at large radii where there is substantial interaction with the external medium.

The study of the early phases of the ejecta interaction with the external medium can probe the strength of magnetic fields in flow. The early emission from the forward shock, for example, depends on the magnetization of the ejecta (Lyutikov \& Blandford 2003; Zhang \& Kobayashi 2005, hereafter ZK05; Genet et al. 2006). Modeling of the microphysical parameters of the forward and the reverse shock supports the existence of strong magnetic fields in the ejecta (Fan et al. 2002; Kumar \& Panaitescu 2003; Zhang et al. 2003). Furthermore, polarization measurements can provide information on the structure of the magnetic fields (Lazzati et al. 2003; Granot \& Taylor 2005; Mundell et al. 2007).

The early optical afterglow lightcurves depend critically on the very existence of a reverse shock into strongly magnetized ejecta. A reverse shock may also contribute in a dominant way to the observed emission in later afterglow phases (Genet et al. 2007; Uhm \& Beloborodov 2007). In this paper, we investigate the parameter space of the magnetization of the flow for which there is a reverse shock crossing the ejecta. This parameter space is described by simple analytic expressions. We show that, in contrast to previous claims, if the flow contains similar amounts of kinetic and magnetic energy at the onset of the afterglow, there is no reverse shock forming in a large fraction of GRBs. The observational implications of our findings are also discussed.

\section{The early afterglow phases}

\subsection{Magnetization of the flow at the onset of the afterglow}

For the GRB flow to be accelerated to ultrarelativistic speeds, it must be launched with a high energy-to-rest-mass ratio. The primary form of the energy of the outflow remains unknown. In the fireball model (Goodman 1986; Paczynski 1986), most of the energy is assumed to be initially stored in thermal form while in PDFs (Usov 1992; Mészáros \& Rees 1997b; Spruit et al. 2001; Van Puten \& Ostriker 2001; Uzdensky \& MacFadyen 2006) in magnetic energy density.

Neutrino-antineutrino annihilation taking place in the polar region of a hyper-accreting central object can lead to the formation of a fireball (e.g., Woosley 1993; Ruffert \& Janka 1998; Aloy et al. 2000; Aloy et al. 2005). The fireball is accelerated by its internal pressure gradients that convert its thermal energy into kinetic energy of the baryons. After the acceleration phase is over, the flow may consist of faster and slower shells that collide with each other. These collisions lead to internal shocks that power the prompt emission (Paczynski \& Xu 1994; Rees \& Mészáros 1994; Daigne \& Mochkovitch 1998; Mimica et al. 2005; Mimica et al. 2007a). Internal shocks can lead to 
amplification of seed magnetic fields due to, e.g., the two-stream instability (Medvedev \& Loeb 1999). The strength of these small scale fields and their survival on macroscopic scales downstream of the shocks is rather uncertain (e.g. Spitkovsky 2007). Still, they may account for $\sim 0.1-1 \%$ of the luminosity of the flow. After the phase of internal collisions, the flow expands and cools before it enters the afterglow phase. There, the flow is expected to be weakly magnetized (i.e. the magnetic energy density is much less than the rest mass energy density of the flow).

On the other hand, magnetic fields can be efficient in extracting rotational energy from the central engine which may be an accretion disk (Blandford \& Payne 1982), a rotating black hole (Blandford \& Znajek 1977) or a millisecond magnetar (Usov 1992). The resulting flow is initially Poynting-flux dominated. The acceleration of a PDF is more model dependent than that of the fireball model. It depends on the magnetic field geometry and on the role of magnetic dissipation. In particular, magnetic dissipation can lead to efficient acceleration of the flow by converting Poynting flux in to kinetic energy (Drenkhahn \& Spruit 2002; Giannios \& Spruit 2006). Magnetic dissipation can also efficiently power the prompt emission (Thompson 1994; Spruit et al. 2001; Lyutikov \& Blandford 2003; Giannios 2006b; Thompson 2006; Giannios \& Spruit 2007). Despite the model dependent aspects of MHD jet acceleration, a common outcome of different studies is that the acceleration process is not $100 \%$ efficient in converting the Poynting flux into kinetic flux. As a result, the flow remains strongly magnetized after the acceleration phase is over. At large radii, magnetic fields carry energy comparable to that of the kinetic energy of the baryons ( $\mathrm{Li}$ et al. 1992; Drenkhahn \& Spruit 2002; Vlahakis \& Königl 2003; Giannios \& Spruit 2006) or even dominate over the kinetic energy of the flow (e.g. Lyutikov \& Blandford 2003; Thompson 2006; Lyutikov 2006).

It has to be noted that the degree of magnetization of the ejecta may critically depend on the likelihood of baryon mixing in the course of the generation of a PDF, which can only be reliably determined by extremely well resolved MHD numerical simulations. A substantial baryon entrainment could result in a much smaller magnetization than predicted by the above mentioned models. Furthermore, the magnetization of PDFs could be substantially decreased by the pair-loading resulting from $v \bar{v}$ annihilation close to the central engine (see, e.g., Levinson \& Eichler 1993; Aloy \& Obergaulinger 2007).

\subsection{Initial interaction of the ejecta with the external medium}

If far enough away from the central engine, there is substantial interaction of the relativistic ejecta with the external medium. This interaction is believed to result to the afterglow emission. A characteristic difference between fireballs and PDFs lies in the magnetization of the ejecta at the onset of the afterglow phase. In fireball models, the energy of the flow is dominated by the kinetic energy of baryons. If the flow is launched Poynting-flux dominated, it is expected to maintain a large fraction of its energy in the form of magnetic energy at large distance.

Since the initial phases of the ejecta-external medium interaction depend on the magnetization of the ejecta (e.g., Lyutikov 2006), it is possible distinguish among fireball and PDF models using early afterglow observations. A particularly promising probe of the magnetic content of the flow comes from the study of the reverse shock emission (e.g. Kumar \& Panaitescu 2003; Zhang et al. 2003). In this work, we focus on the conditions for the existence of a reverse shock in arbitrarily magnetized ejecta.
We focus on the interaction of the GRB ejecta with external medium of constant number density $n_{\mathrm{e}}$. Similar analysis can, however, be applied to different density profiles for the external medium. The case of wind-like density profile $n \sim r^{-2}$ is also discussed in Sect. 3.2. For typical GRB parameters, this interaction becomes substantial at distances $r \sim 10^{15}-10^{17} \mathrm{~cm}$. We assume that the acceleration and collimation of the flow has taken place much closer to the central engine as well as the internal dissipation mechanisms (e.g. internal shocks, magnetic dissipation) that are believed to power the prompt emission. After the internal dissipation is over, the flow expands and cools down. Since we are interested in the afterglow phase, we consider a, radially moving, cold and relativistic shell with width $\Delta_{0}$ and corresponding Lorentz factor $\gamma_{0} \gg 1$.

In a radially moving magnetized flow, the induction equation results in a component of the magnetic field perpendicular to the motion $B_{\perp} \propto 1 / r$ that drops much slower with radius than the parallel one: $B_{\|} \propto 1 / r^{2}$. Since the flow expands for several order of magnitude from the launching to the afterglow region, we assume that the magnetic field is dominated by its perpendicular component. The magnetic content of the shell is conveniently parameterized by its magnetization $\sigma_{0}$ which stands for the Poynting-to-kinetic flux ratio at some initial radius $r_{0}$. For an ultrarelativistic flow

$\sigma_{0} \equiv \frac{E_{\mathrm{p}}}{E_{\mathrm{k}}}=\frac{B_{0}^{2}}{4 \pi \gamma_{0} \rho_{0} c^{2}}$,

where $B_{0}$ and $\rho_{0}$ are the initial magnetic field strength and density of the shell; both measured in the lab frame. Note that, since the shell is assumed initially cold, we neglect the contribution of the internal energy to the kinetic flux of the shell.

The total energy of the shell is

$E=4 \pi R_{0}^{2} \Delta_{0}\left(\gamma_{0} \rho_{0} c^{2}+B_{0}^{2} / 4 \pi\right)=E_{\mathrm{k}}\left(1+\sigma_{0}\right)$

where the first term stands for the kinetic energy and the second for the magnetic energy of the shell. In other words, the magnetization $\sigma_{0}$ parameterizes the fraction of the total energy of the flow that is in kinetic and in magnetic form. These fractions are $1 /\left(1+\sigma_{0}\right)$ and $\sigma_{0} /\left(1+\sigma_{0}\right)$ respectively.

Once $E, \Delta_{0}, \gamma_{0}$ and $\sigma_{0}$ are fixed the properties of the ejecta are defined. The reference values that we adopt for these parameters are summarized in the following. In our spherically symmetric model $E$ is roughly equal to the isotropic equivalent energy $E_{\text {iso }}$ of the burst and, thus $E \sim E_{\text {iso }} \sim 10^{53} E_{53} \operatorname{erg}$ (with spread of a couple of orders of magnitude from burst to burst). The width of the shell can be related to the observed duration $T_{\mathrm{GRB}}$ of the GRB through the relation $\Delta_{0}=c T_{\mathrm{GRB}} \sim 10^{12} \Delta_{12} \mathrm{~cm}$ for a $\sim 30 \mathrm{~s}$ burst. This connection between observed burst duration and shell width holds in the internal shock model (Rees \& Mészáros 1994; Nakar \& Piran 2002) and photospheric models (as discussed, for example, in Giannios \& Spruit 2007) for the prompt emission. The Lorentz factor of the flow is less constrained. From theoretical arguments related to the "compactness problem", it is expected to be $\gamma_{0} \gtrsim 100$. We adopt $\gamma_{0}=10^{2.5} \gamma_{2.5}$ as reference value. Among the shell parameters, $\sigma_{0}$ is the most model dependent quantity and we deal with it as a free parameter. In fireball models $\sigma_{0} \ll 1$ while in PDFs $\sigma_{0} \gtrsim 1$.

Before studying the initial interaction of arbitrarily magnetized ejecta with the external medium, we summarize the results of the unmagnetized $\sigma_{0}=0$ case. These results facilitate the presentation of the more general case where $\sigma_{0}$ is arbitrary. 


\subsubsection{Non-magnetized ejecta}

The deceleration of non-magnetized ejecta has been well studied with both analytical (Sari \& Piran 1995; Mészáros \& Rees 1997a) and numerical approaches with 1-dimensional (Kobayashi et al. 1999) and 2-dimensional (Granot et al. 2001; Cannizzo et al. 2004; Meliani et al. 2007) hydrodynamical simulations. The 2-dimensional studies are important to follow the late-time lateral spreading of collimated ejecta. Here, we are interested in the initial phases of the deceleration of the ejecta. These can be followed assuming spherical symmetry since the initial Lorentz factor of the flow is likely larger than the inverse of the jet opening angle and, therefore, lateral spreading is unimportant.

The hydrodynamical evolution of the shell-external medium configuration is rather well understood. The interaction of the two media leads to a forward shock that propagates into the external medium. At the same time a second shock (the so called reverse shock) propagates into the ejecta. The formation of the reverse shock always takes place in cold, non-magnetized ejecta since the shocked material moves supersonically with respect to the unshocked shell. This can be formally shown by solving the initial Riemann problem at the ejecta-external medium interface (see, for example Rezzolla \& Zanotti 2001). We will see that this is not always the case in strongly magnetized ejecta where MHD waves can transfer information at relativistic speeds. The shell can, thus, smoothly adjust its properties upon interacting with the external medium.

The strength of the reverse shock depends on the ratio of the densities of the shell and the external medium and on the bulk Lorentz factor of the flow. It can be shown (Sari \& Piran 1995) that the strength of the reverse shock can be conveniently parameterized by

$\xi \equiv \sqrt{\frac{l}{\Delta_{0}}} \frac{1}{\gamma_{0}^{4 / 3}}$,

where $l=\left(3 E / 4 \pi n_{\mathrm{e}} m_{\mathrm{p}} c^{2}\right)^{1 / 3}$ is the Sedov length. In the limit where $\xi \gg 1$ the reverse shock is Newtonian and, if $\xi \ll 1$, relativistic (Sari \& Piran 1995).

The $\xi$ quantity can be related to the more familiar "deceleration radius" $r_{\mathrm{dec}}$ and the "spreading radius" $r_{\mathrm{s}}$. The first is defined as the radius where the ejecta accumulate from the external medium a mass $\gamma_{0}^{-1}$ times their own mass, resulting in $r_{\mathrm{dec}}=l / \gamma_{0}^{2 / 3}$. The $r_{\mathrm{s}}$ is the radius where a shell with mildly relativistic proper motions starts spreading radially $r_{\mathrm{s}}=\Delta_{0} \gamma_{0}^{2}$ (see Sect. 3.1). Using the last expressions, $\xi$ can be expressed as

$\xi=\sqrt{\frac{r_{\mathrm{dec}}}{r_{\mathrm{s}}}}$.

In terms of $r_{\mathrm{dec}}$ and $r_{\mathrm{s}}$, the condition for a relativistic reverse shock is that $r_{\mathrm{dec}} \ll r_{\mathrm{s}}$.

Using as reference value for the external medium number density $n_{\mathrm{e}}=10^{0} n_{0} \mathrm{~cm}^{-3}$ we have that

$\xi=0.73 \frac{E_{53}^{1 / 6}}{n_{0}^{1 / 6} \Delta_{12}^{1 / 2} \gamma_{2.5}^{4 / 3}}$,

showing that $\xi$ possesses a very weak dependence with $E_{53}$ and $n_{0}$. For various GRB parameters $\xi$ can typically vary in the $0.1 \lesssim$ $\xi \lesssim 10$ range allowing for both Newtonian and relativistic reverse shocks in different bursts.
In the Newtonian reverse shock limit, the ejecta do not decelerate much during the reverse shock crossing. They enter the deceleration phase once they accumulate from the external medium mass that is a factor $\gamma_{0}$ smaller than their own mass. In the relativistic reverse shock case substantial deceleration takes place already when the reverse shock crosses the ejecta. In both relativistic and Newtonian reverse shock limits the radius of the reverse shock crossing $r_{\mathrm{rs}}^{\mathrm{H}}$ is given by the expression ${ }^{1}$ (Sari \& Piran 1995)

$r_{\mathrm{rs}}^{\mathrm{H}}=l^{3 / 4} \Delta_{0}^{1 / 4}=\left(r_{\mathrm{s}} r_{\mathrm{dec}}^{3}\right)^{1 / 4}$

where the index " $\mathrm{H}$ " stands for hydrodynamical (nonmagnetized) case.

In the Newtonian reverse shock case, the radius of the reverse shock crossing is modified if one allows for mildly relativistic relative speeds within the shell. The relative motions lead to shell spreading and strengthening of the reverse shock. This effect is considered in Sect. 3.1

After the reverse shock reaches the back part of the ejecta, there is an initial phase of interaction where shocks and rarefaction waves cross the ejecta. Gradually most of the energy is passed in the shocked external medium and the whole structure relaxes to the self-similar structure described in Blandford $\&$ McKee (1976). From this point on, the evolution of the blastwave depends only on the energy $E$ and the density of the external medium $n_{\mathrm{e}}$.

\subsubsection{Arbitrarily magnetized ejecta}

The dynamics of the deceleration of magnetized ejecta has not been studied in the same detail. To understand the various phases of the deceleration of the ejecta and the transfer of their energy into the shocked external medium one needs to follow the dynamics of ejecta-external medium interaction in detail. The internal evolution of the shell is particularly important in this respect. The study of the complete deceleration dynamics is the goal of the relativistic MHD simulations to be reported elsewhere (Mimica et al. 2007b). Here we are concerned with the very initial phase of the interaction where the reverse shock is (or is not) crossing the ejecta and derive the conditions for such shock to form.

The initial phase of reverse shock crossing has been studied by Zhang \& Kobayashi (2005) by solving the ideal MHD shock conditions for arbitrarily magnetized ejecta with a dominant toroidal field (see also Fan et al. 2004 for the case of mildly magnetized ejecta). Their analysis describes the reverse shock crossing phase provided that there is a reverse shock forming. They show that the reverse shock crosses faster ejecta of high magnetization $\sigma_{0}$ with respect to un-magnetized ejecta of the same $E, \Delta_{0}, \gamma_{0}$. They show that the reverse shock crossing radius scales as $r_{\mathrm{rs}} \propto 1 / \sqrt{\sigma_{0}}$ for $\sigma_{0} \gg 1$ and can be written

$r_{\mathrm{rs}}=l^{3 / 4} \Delta_{0}^{1 / 4} C_{\Delta}=\left(r_{\mathrm{s}} r_{\mathrm{dec}}^{3}\right)^{1 / 4} C_{\Delta}$.

The coefficient $C_{\Delta}$ depends mainly on the magnetization $\sigma_{0}$ and very weakly on the relative Lorentz factor of the shockedunshocked ejecta (see Fig. 3 in ZK05). For the purposes of this work, we adopt the expression $C_{\Delta} \sim 1 / \sqrt{1+\sigma_{0}}$ which is rather accurate in reproducing their results. Note that this expression for $C_{\Delta}$ gives also the correct zero magnetization limit (6).

1 The reverse crossing radius differs in the two limits by a small factor which we ignore here. 


\section{Condition for existence of a reverse shock}

Cold, non-magnetized ejecta are always crossed by a reverse shock upon interacting with the external medium. This is the case since the sound speed of the ejecta is low and does not allow for fast transfer of the information of the interaction with the external medium throughout their volume. On the other hand, in a flow that is strongly magnetized and sub-fast magnetosonic (as in the Lyutikov \& Blandford 2003, model) there is no reverse shock forming. The flow adjusts gradually to the changes of the pressure in the contact discontinuity that separates the magnetized flow from the shocked external medium. Here we generalize to arbitrarily magnetized ejecta and derive the condition for the formation of a reverse shock.

An important radius to be considered for the existence of a reverse shock is the "contact" radius $r_{\mathrm{c}}$ at which fast MHD waves cross the width of the shell. At this radius, the information on the conditions of the front part has been transfered to the rest of the shell. After this radius is crossed the magnetized shell comes in pressure balance and adjusts its structure on a timescale shorter than the expansion time. Any gradual changes of the physical properties at the contact discontinuity that separates the shell from the shocked external medium lead to smooth evolution of the shell properties. The very existence of the contact radius implies that different parts of the flow have dropped out of MHD contact during the acceleration phase. Our analysis applies for super-fast magnetosonic flows (i.e. flows with $\gamma_{0}>\sqrt{1+\sigma_{0}}$ ) for which this is the case.

A second important radius for the existence of a reverse shock is $r_{\mathrm{rs}}$ (see Eq. (7)). If $r_{\mathrm{c}}>r_{\mathrm{rs}}$, a reverse shock forms and crosses the ejecta before MHD contact within the shell is established. In this case, internal evolution does not take place in the shell before the reverse shock crossing. On the other hand, if $r_{\mathrm{c}}<r_{\mathrm{rs}}$, there is no reverse shock forming and $r_{\mathrm{rs}}$ does not have a physical meaning. The condition for the formation of a reverse shock is, thus, to set $r_{\mathrm{c}}>r_{\mathrm{rs}}$. Note that this condition states the obvious fact that there is a reverse shock forming as long as it propagates supersonically into the ejecta. As we discuss in Sect. 3.3, a different condition for the existence of a reverse shock adopted by ZK05 leads to incorrect estimates on the magnetization needed to suppress the formation of a reverse shock.

The "contact" radius is given by the distance the shell travels before a fast-magnetosonic wave crosses the shell. Any news from the front travels into the shell with speed $\beta_{A}=$ $\sqrt{\sigma_{0} /\left(1+\sigma_{0}\right)}$ (with a corresponding Lorentz factor $\gamma_{A}=$ $\left.\sqrt{1+\sigma_{0}}\right)$ in the frame comoving with the shell. As seen in the central engine frame, this wave moves with Lorentz factor $\gamma_{\mathrm{w}}$ given by the expression $\gamma_{A} \approx\left(\gamma_{\mathrm{w}} / \gamma_{0}+\gamma_{0} / \gamma_{\mathrm{w}}\right) / 2$. The last expression is accurate for $\gamma_{0}, \gamma_{\mathrm{w}} \gg 1$ and can be solved for $\gamma_{\mathrm{w}}$ resulting in $\gamma_{\mathrm{w}}=\gamma_{0}\left(\sqrt{1+\sigma_{0}}-\sqrt{\sigma_{0}}\right)$. The shell comes into contact when the wave crosses it. This takes place at radius

$r_{\mathrm{c}}=\frac{\Delta_{0} c}{v_{0}-v_{\mathrm{w}}} \simeq \Delta_{0} \gamma_{0}^{2}\left(\sqrt{\frac{1+\sigma_{0}}{\sigma_{0}}}-1\right)$

where we have used that $v_{\mathrm{w}} / c \simeq 1-1 / 2 \gamma_{\mathrm{w}}^{2}$ and $v_{0} / c \simeq 1-1 / 2 \gamma_{0}^{2}$.

The contact radius depends only on the shell properties and not on those of the external medium. The reason is that the speed of propagation of fast-magnetosonic waves depends only on the magnetization of the shell as a function of the radius $\sigma(r)$ which turns out to be equal to the initial value $\sigma_{0}$. To check such an assertion, one needs to consider that a shell that expands spherically decreases both its density and its magnetic energy density

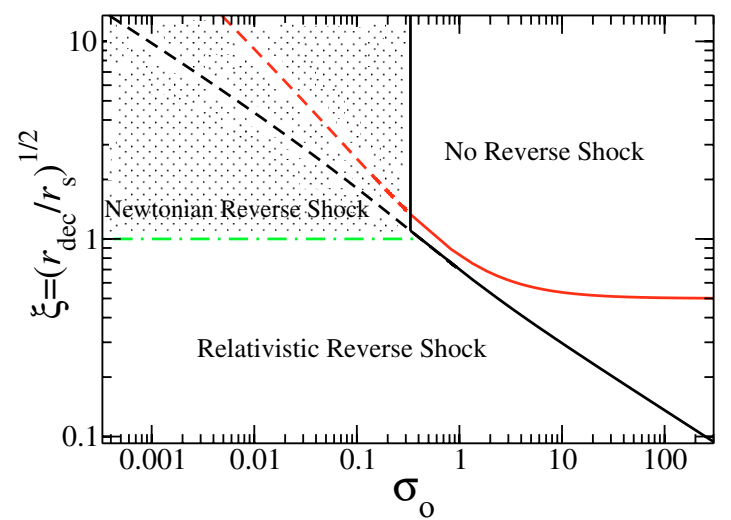

Fig. 1. Regime of existence of a reverse shock in the $\xi$ - $\sigma_{0}$ plane. The $\xi$ is defined by Eq. (3) (or Eq. (4)) and $\sigma_{0}$ stands for the Poynting-tokinetic flux ratio of the flow at the onset of the afterglow. Ignoring radial spreading of the ejecta, the lower left part with respect to the black, dashed line shows the regime where there is a reverse shock forming for a constant-density external medium. For $\sigma_{0} \gtrsim 1$ there is no reverse shock forming for a large parameter space that describes the properties of the GRB ejecta. The solid line shows the frontier between "no reverse shock" and "reverse shock" when radial spreading of the shell is taken into account. In the case of shell spreading, the shaded area is characterized by a mildly relativistic reverse shock (see Sect. 3.1). Similarly, the red lines show the frontier between "no reverse shock" and "reverse shock" in the wind-like external medium.

as $r^{-2}$. Therefore, $\sigma(r)$ remains uniform in the shell and equal to its initial value (Eq. (1)) until, at least, the shell reaches $r_{\mathrm{c}}$.

From Eq. (8) we see that in the limit of $\sigma_{0} \gg 1, r_{\mathrm{c}} \simeq$ $\Delta_{0} \gamma_{0}^{2} / 2 \sigma_{0} \propto \sigma_{0}^{-1}$ while for $\sigma_{0} \rightarrow 0, r_{\mathrm{c}} \rightarrow \infty$. In the hydrodynamical $\left(\sigma_{0}=0\right)$ limit $r_{\mathrm{c}}>r_{\mathrm{rs}}$ and a reverse shock crosses the ejecta at $r_{\text {rs. }}$. On the other hand, since the contact radius scales with the magnetization as $r_{\mathrm{c}} \propto \sigma_{0}^{-1}$, it is reduced faster than the $r_{\mathrm{rs}} \propto \sigma_{0}^{-1 / 2}$ for increasing $\sigma_{0}$. There is always a magnetization for which $r_{\mathrm{rs}}$ and $r_{\mathrm{c}}$ become equal and the reverse shock is suppressed.

The condition for the existence of the reverse shock is given by setting $r_{\mathrm{rs}}<r_{\mathrm{c}}$ and using Eqs. (7), (8) and the definition (3) of $\xi$. The line that separates the reverse shock from the non-reverse shock regime in the $\xi-\sigma_{0}$ parameter space is

$\left(\frac{r_{\mathrm{dec}}}{r_{\mathrm{s}}}\right)^{3 / 2}=\xi^{3}=\frac{1+\sigma_{0}}{\sigma_{0}}\left(1+2 \sigma_{0}-2 \sqrt{\sigma_{0}\left(1+\sigma_{0}\right)}\right)$.

In the limit of small and large $\sigma_{o}$ the last expression simplifies respectively to

$\xi=\frac{1}{\sigma_{0}^{1 / 3}}$ for $\sigma_{0} \ll 1$,

$\xi=\frac{1}{\left(4 \sigma_{0}\right)^{1 / 3}} \quad$ for $\quad \sigma_{0} \gg 1$.

Although, the expression $\xi=\left(4 \sigma_{0}\right)^{-1 / 3}$ is formally derived for $\sigma_{0} \gg 1$, it is still a fair approximation for $\sigma_{0} \gtrsim 0.1$ and will be used in the following discussion. Solving the last expression for $\sigma_{0}$, and using the reference values of the GRB parameters we find that a reverse shock forms when

$\sigma_{0} \lesssim 0.6 n_{0}^{1 / 2} \Delta_{12}^{3 / 2} \gamma_{2.5}^{4} E_{53}^{-1 / 2}$

The curve defined by Eq. (9) is shown in Fig. 1 with a dashed line that turns into solid for $\sigma_{0} \gtrsim 0.3$. It separates the $\xi-\sigma_{0}$ parameter space into regions with the upper right one characterized 
by no reverse shock. In the $\sigma_{0} \ll 1$ regime the $\xi=1$ dash-dotted line separates the Newtonian reverse shock $(\xi>1)$ regime from the $\xi<1$ relativistic reverse shock one. Note that a small magnetization suffices to suppress a reverse shock for large $\xi$ and vice versa.

As it is obvious from Eq. (11), for typical GRB parameters for a strongly magnetized flow with $\sigma_{0} \gtrsim 1$ there is no reverse shock forming. In this case, the onset of the afterglow emission will not be characterized by an optical flash (Mészáros \& Rees 1997a; Sari \& Piran 1999) that is expected to be the signature of the reverse shock emission. Note, however that this conclusion depends sensitively on some of the GRB parameters. This is especially true for the Lorentz factor of the GRB flow which is also, perhaps, the least constrained parameter. Had we used a reference value of $\gamma_{0}=1000$ for it then the transition to no reverse shock would take place for $\sigma_{0} \sim$ a few tens. This means that one can still have a reverse shock forming in a $\gamma_{0} \gtrsim 1000$, $\sigma_{0} \gg 1$ flow. However, when a reverse shock forms in a high $\sigma_{0}$ flow it is not expected to result in bright emission (at least in the ideal MHD limit discussed here) since the shock dissipates only part of the kinetic energy which is a small fraction of the total energy of the shell (ZK05).

So far, we have focused in ejecta with initially uniform motion, i.e. with no radial spreading. Shell spreading introduces new features to the picture presented so far. These features are the topic of the next section.

\subsection{Shell-spreading effects}

It is quite likely that the ejecta are characterized by relative motions after the acceleration, collimation and internal interaction phases. These relative motions may well be mildly relativistic in the shell rest frame leading to ejecta with moderate variations of the Lorentz factor $\delta \gamma_{0} \sim \gamma_{0}$ along their width.

Ejecta with their front part moving faster than their rear one with moderate variation of the bulk Lorentz factor start spreading at radius $r_{\mathrm{s}} \simeq \gamma_{0}^{2} \Delta_{0}$ (Sari \& Piran 1995; Kobayashi et al. 1999). Because of the spreading, the width of the shell increases linearly with distance at larger radii. It has to be noted that $\Delta_{0} / c$ does not necessary correspond to the time during which the central engine is actively releasing energy into the outflow. As pointed out by Aloy et al. (2005), in case of short GRBs, the initial shell width $\Delta_{\mathrm{e}}=c T_{\mathrm{a}}\left(T_{\mathrm{a}}\right.$ being the time during which the central engine actively releases energy) may stretch radially by a factor $\sim 2-20$ due to the same mechanism invoked for shell spreading at later times, namely, the relative velocity difference between the forward and rear radial edges of the ejecta.

In the case of non-magnetized ejecta with $\xi>1$, the shell starts spreading before the reverse shock crossing. Radial expansion leads to density decrease in the shell which is faster than $1 / r^{2}$. As a result, by the end of the reverse shock crossing the shock is mildly relativistic. When $\xi<1$, the reverse shock crosses the shell before it reaches the spreading radius $r_{\mathrm{s}}$. The relativistic reverse shock case is, hence, not affected by the spreading of the shell since, by the time the shock has crossed the shell the density of the latter has not dropped appreciably in comparison to the non-spreading case (Sari \& Piran 1995). One may also express the condition of formation of a relativistic reverse shock in terms of the deceleration radius $r_{\mathrm{dec}}$ and $r_{\mathrm{s}}$, which reads $r_{\text {dec }} \ll r_{\mathrm{s}}$.

Spreading of the shell influences the interaction of the ejecta with the external medium for magnetized ejecta as well. If the contact radius $r_{\mathrm{c}}$ is larger than the spreading radius, the shell spreads before fast MHD waves produced in the front part of the shell catch up with the rear part. Using the estimate $r_{\mathrm{s}}=\gamma_{0}^{2} \Delta_{0}$ for the spreading radius and Eq. (8) for $r_{\mathrm{c}}$, one finds that $r_{\mathrm{s}} \lesssim r_{\mathrm{c}}$ for $\sigma_{0} \lesssim 0.3$. For low enough $\sigma_{0}$ the shell does not come into MHD contact because of radial spreading. Under these conditions, a mildly relativistic RS forms just like in the $\sigma_{0}=0$ case.

The shaded region of Fig. 1 shows the regime where spreading of the shell leads to the formation of a reverse shock. Quite generally for $\sigma_{0} \lesssim 0.3$, there is always a reverse shock forming in a shell that spreads because of mildly relativistic proper motions.

\subsection{Deceleration in a wind environment}

A possible environment of the deceleration of the GRB flow is that of stellar wind (i.e. "collapsar" model for long GRBs; Woosley 1993). The density profile of the external medium in this case is $n_{\mathrm{w}}=A / r^{2}$. Here, we derive briefly the condition for the existence of a reverse shock in such density profile.

The reverse-shock crossing radius for un-magnetized ejecta that decelerate in wind density profile is $r_{\mathrm{rs}, \mathrm{w}}^{\mathrm{H}} \sim\left(r_{\mathrm{s}} r_{\mathrm{dec}, \mathrm{w}}\right)^{1 / 2}$ (Sari \& Piran 1995), where the deceleration radius is defined as $r_{\mathrm{dec}, \mathrm{w}}=l_{\mathrm{w}} / \gamma_{0}^{2}=E / 4 \pi A m_{\mathrm{p}} \gamma_{0}^{2} c^{2}$. The $l_{\mathrm{w}}$ is the Sedov length for the wind density profile. If the ejecta is magnetized, the reverse shock crosses them faster with respect to the hydrodynamical case. In the high- $\sigma_{0}$ limit the (reverse) shock conditions lead to $r_{\mathrm{rs}, \mathrm{w}} \simeq r_{\mathrm{rs}, \mathrm{w}}^{\mathrm{H}} / \sigma_{0}(\mathrm{e} . \mathrm{g} . \mathrm{ZK} 05)$. An approximate expression for the radius of the crossing of the reverse shock that reproduces both the $\sigma_{0} \rightarrow 0$ and $\sigma_{0} \gg 1$ limits is

$r_{\mathrm{rs}, \mathrm{w}} \sim \frac{\left(r_{\mathrm{s}} r_{\mathrm{dec}, \mathrm{w}}\right)^{1 / 2}}{1+\sigma_{0}}$.

The condition for existence of a reverse shock is that the $r_{\mathrm{rs}, \mathrm{w}}<$ $r_{\mathrm{c}}$. The contact radius depends only on the shell properties and not on the density profile of the external medium; it is defined by Eq. (8). The line that separates the "no reverse shock" from the "reverse shock" regime is found by setting $r_{\mathrm{rs}, \mathrm{w}}=r_{\mathrm{c}}$. It results in:

$\xi_{\mathrm{w}}=\left(\frac{r_{\mathrm{dec}, \mathrm{w}}}{r_{\mathrm{s}}}\right)^{1 / 2}=\left(1+\sigma_{0}\right)\left(\sqrt{\frac{1+\sigma_{0}}{\sigma_{0}}}-1\right)$.

The line defined by Eq. (13) is shown in the $\xi$ - $\sigma_{0}$ plane of Fig. 1.

If the shell is characterized by mildly relativistic proper motions, its spreading radius is $r_{\mathrm{s}} \sim \Delta_{0} \gamma_{0}^{2}$. Just as in the case of constant density external medium, spreading of the shell leads to the formation of a reverse shock when $\sigma_{0} \lesssim 0.3$.

\subsection{Non-ideal MHD effects}

In this work, we focus on the existence of a reverse shock (and the associated emission) coming from the interaction of a shell, permitted by a large scale magnetic field, with the external medium. Furthermore, this analysis is based on the ideal MHD shock conditions derived by ZK05. However, non-ideal MHD effects may also be of relevance in the flow.

It is possible, for example, that the magnetized flow is launched with magnetic fields that contain a random component; one that changes polarity on short scales in the radial direction (Thompson 1994; Drenkhahn \& Spruit 2002; Thompson 2006). Furthermore small scale field reversals may develop during the acceleration phases as result of MHD instabilities in the jet (Giannios \& Spruit 2006). Unless magnetic reconnection is efficient in dissipating the random component of the magnetic field (e.g. Drenkhahn \& Spruit 2002), small scale reversals of 
the field along the radial direction are still present in the afterglow regime. Such field structure can have an effect on the shock conditions and therefore on the condition for the existence of a reverse shock (for discussion see Thompson 2006).

Dissipation of part of the magnetic energy that is contained in the flow at large radii can result to particle acceleration and emission with a variety of observational consequences. This emission is not directly related to shock-accelerated particles. The reverse shock can, for example, lead to forced reconnection of a reversing magnetic field. The released energy can power the prompt GRB emission (Thompson 2006). Magnetic dissipation can also take place during a later stage of the deceleration of the ejecta. In this picture, slowing down of the ejecta leads to the revival of MHD instabilities in regions of the flow that come into causal contact again. Such delayed dissipation can power the afterglow flares (Giannios 2006a) observed in many early X-ray afterglows (Burrows et al. 2005; Zhang et al. 2006).

\subsection{Comparison with previous work}

The early phases of interaction of the GRB ejecta have been previously studied in the hydrodynamical limit (i.e. $\sigma_{0} \rightarrow 0$; Sari \& Piran 1995) and for a sub-fast magnetosonic, strongly magnetized, flow (i.e. $\gamma_{0}^{2}<1+\sigma_{0}$; Lyutikov \& Blandford 2003; Genet et al. 2006). The outcome of those studies is that in the former limit there is always a reverse shock developing, in the latter the flow adjusts smoothly to the properties of the shocked external medium and there is no reverse shock. Those results are in agreement with these reported here.

The intermediate range of magnetization has been studied in ZK05 by solving for the shock conditions for the forward and the reverse shock. Their study is applicable for the parameter space where there is a reverse shock forming. They also estimate the conditions for the formation of a reverse shock assuming a constant density external medium. They find that large values of $\sigma_{0} \sim 100$ are needed (for the reference GRB parameters) to suppress the existence of a reverse shock. This estimate is more than two orders of magnitude different from the one presented here. To probe the difference, we briefly sketch the ZK05 condition for the existence of a reverse shock.

$\mathrm{ZK} 05$ define the deceleration radius as the radius where the ejecta accumulate from the external medium mass a factor $1 / \gamma_{0}$ less than the external medium mass: $r_{\mathrm{d}}^{\mathrm{ZK}}=l / \gamma_{0}^{2 / 3}\left(1+\sigma_{0}\right)^{1 / 3}$. The conditions for the existence of a reverse shock that they use are that (i) the flow speed exceeds the fast magnetosonic speed (i.e. $\gamma_{0}>\sqrt{1+\sigma_{0}}$ ) and (ii) the magnetic pressure of the shell is lower than that of the shocked external medium at the deceleration radius $r_{\mathrm{d}}^{\mathrm{ZK}}$ : i.e. that $B_{0}^{2} / 8 \pi \gamma_{0}^{2}<(4 / 3) \gamma_{0}^{2} \rho_{\mathrm{e}} c^{2}$. In the last expression, one uses that the comoving magnetic field is $B_{0 \text {,co }}=B_{0} / \gamma_{0}$ and the forward relativistic shock condition that gives for the gas pressure of the shocked external medium: $P_{\mathrm{g}}=(4 / 3) \gamma_{0}^{2} \rho_{\mathrm{e}} c^{2}$. Using Eq. (2) and focusing in the $\sigma_{0} \gg 1$ limit, the condition for $\sigma_{0}$ for the existence of a reverse shock can be written $\sigma_{0} \lesssim 190 / \xi^{3}$. The boundary that separates the shock from the non-shock regime is $\sigma_{\mathrm{ZK}} \simeq 190 / \xi^{3}$. It is very different with respect to that of Eq. (10) derived here.

What is the source of this discrepancy? ZK05 apply their condition in the deceleration radius and not the reverse shock crossing radius which we believe is the relevant one so as to check the existence of a reverse shock ${ }^{2}$. Furthermore, the condition itself for the existence of the reverse shock used in ZK05

2 Note also that the definition of the deceleration radius is controversial (see Lyutikov's 2005 comments). is questionable. For magnetization $\sigma_{0} \sim \sigma_{\mathrm{ZK}}$ the "contact" radius $r_{\mathrm{c}}$ is much shorter than the "deceleration" radius $r_{\mathrm{d}}^{\mathrm{ZK}}$ and the reverse shock crossing radius $r_{\mathrm{rs}}$. This means that one gets the erroneous result that the reverse shock moves subsonically with respect to the unshocked material. The fact that $r_{\mathrm{c}}<r_{\mathrm{d}}^{\mathrm{ZK}}$ indicates instead that much before the flow reaches $r_{\mathrm{d}}^{\mathrm{ZK}}$ the shell has already evolved. The spherical geometry starts to play an important role and the problem becomes that of dynamical evolution of the shell properties and not just shock conditions of a non evolving shell.

On the other hand, when a reverse shock does form then it crosses the shell before its different parts have time to come into contact through exchange of MHD waves. This means that the properties of the shell upstream to the shock do not evolve much before the shock crossing. In this case the analysis of ZK05 is applicable. This justifies the use of their result on the reverse shock crossing radius given by Eq. (7).

\section{Discussion}

The role of magnetic fields in GRB flows remains uncertain. In fireball models, the GRB flow is launched with dynamically unimportant magnetic fields while MHD energy extraction can lead to a Poynting-flux dominated flow. Observations of the prompt emission alone appear hard to distinguish between fireball or PDF models ${ }^{3}$. Early afterglow observations can, however, probe the magnetic content of the ejecta. The magnetization can leave its signature in the early forward shock (Lyutikov \& Blandford 2003; Genet et al. 2006) and the reverse shock emission (Kumar \& Panaitescu 2003; Zhang et al. 2003; Fan et al. 2004; ZK05).

\subsection{Magnetization and existence of a reverse shock}

Here, we derive the condition for the existence of a reverse shock in the ejecta that contain a large scale magnetic field, assuming the ideal MHD description is adequate. The ejecta decelerate interacting with the circumburst medium. External medium with both constant and wind-like density profiles are considered. It is shown that, for high enough magnetization, the ejecta interact smoothly with the external medium and decelerate without the formation of a reverse shock. The conditions for the formation of a reverse shock are derived by demanding that the reverse shock propagates supersonically in the ejecta. The conditions are summarized by Eqs. (9) and (11) and Fig. 1. Our results are substantially different from those of ZK05 who do not consider the internal evolution of the ejecta when deriving their criterion for the existence of a reverse shock.

One important finding of the study is that $\sigma_{0} \gtrsim 1$ suffices to suppress the formation of a reverse shock for a large parameter space that characterizes GRB flows. This parameter space is larger for a constant density external medium in comparison to the wind case. This means that only a small fraction of PDFs are expected to show reverse shock emission. Even when a reverse shock forms in a high $\sigma_{0}$ flow, it is not expected to result in bright emission (at least in the ideal MHD limit discussed here) since the shock dissipates only part of the kinetic energy which is a small fraction of the total energy of the shell (see, e.g., ZK05). On the other hand, allowing for radial spreading of the ejecta,

\footnotetext{
3 With the possible exception of multi GeV prompt emission that may show signature of pair cascades in a neutron rich fireball that is absent in PDF flows; Koers \& Giannios (2007).
} 
practically all the flows with $\sigma_{0} \lesssim 0.3$ are expected to be crossed by a mildly-relativistic or relativistic reverse shock.

This work contributes in understanding the early deceleration dynamics of magnetized ejecta. The complete evolution of the system is not considered here. Important questions related to the timescale over which the magnetic energy of the shell (which may dominate the total energy budget) is passed into the shocked external medium remain unexplored. We are currently attacking these questions through relativistic MHD simulations to be reported elsewhere (Mimica et al. 2007b).

\subsection{Connection to observations}

A clear prediction of the fireball models is that there is a reverse shock crossing the ejecta on the onset of the interaction of the ejecta with the external medium. The reverse shock emission is expected to result in a short lived, bright emission event. Although optical flashes have been observed in a few bursts (e.g. GRB 990123, Akerlof et al. 1999; GRB 021211, Fox et al. 2003) and are believed to be powered by the reverse shock emission, they are rare events. This may be a result of a more luminous forward shock masking the reverse shock optical emission (McMahon et al. 2006; Mundell et al. 2007b).

Our analysis points to a different possibility, i.e. that the paucity of bursts with clear reverse shock emission is a result of the frequent reverse shock suppression. It is shown here that this happens for a large GRB parameter space in a strongly magnetized flow. Moreover, in cases where reverse shock emission is identified, modeling of the microphysical parameter connected to the magnetic field strength indicates ejecta with dynamically important magnetic fields (Fan et al. 2002; Kumar \& Panaitescu 2003; Zhang et al. 2003; ZK05) in agreement with our suggestion.

Polarized emission, result of synchrotron emitting, (reverse) shock-accelerated particles is expected from a coherent magnetic field. Note, however, that the lack of significant optical polarization in the early afterglow does not necessarily show the absence of large scale field in the ejecta but may be result of the absence of reverse shock (suppressed by the strong field). This is particularity likely if there is no evidence for the distinct emission patterns expected from a reverse shock. In light of these considerations the claim of Mundell et al. (2007a) that the low early polarization signal in GRB060418 rules out the presence of large scale magnetic field in the ejecta is premature.

This study has focused in the ideal MHD limit. On the other hand non-ideal MHD effects can lead to additional energy release and emission during the afterglow phases of strongly magnetized flows. This emission is not directly related to shock accelerated particles and therefore to the existence or not of a reverse shock. On the other hand, the crossing of the reverse shock can lead to forced reconnection in the ejecta that powers the GRB emission (Thompson 2006). Furthermore, the deceleration of the ejecta can lead to revival of MHD instabilities that lead to magnetic dissipation. Such late-time energy release can power the afterglow flares observed in many early X-ray afterglows (Giannios 2006a).

Acknowledgements. D.G. thanks Henk Spruit for valuable discussions. P.M. is at the University of Valencia with a European Union Marie Curie Incoming International Fellowship (MEIF-CT-2005-021603). M.A.A. is a Ramón y Cajal
Fellow of the Spanish Ministry of Education and Science. He also acknowledges partial support from the Spanish Ministry of Education and Science (AYA200408067-C03-C01).

\section{References}

Akerlof, C., Balsano, R., Barthelmy, S., et al. 1999, Nature, 398, 400

Aloy, M. A., \& Obergaulinger, M., 2007, Rev. Mex. Astron. Astrofís. Conf. Ser., 30, 96, [arXiv:astro-ph/0701187]

Aloy, M. A., Müller, E., Ibáñez, J. M., Martí, J. M., \& MacFadyen, A., 2000, ApJ, 531, L119

Aloy, M. A., Janka, H.-Th., \& Müller, E, 2005, A\&A, 436, 273

Blandford, R. D., \& McKee, C. F. 1976, Phys. Fluids, 19, 1130

Blandford, R. D., \& Payne, D. G. 1982, MNRAS, 199, 883

Blandford, R. D., \& Znajek, R. L. 1977, MNRAS, 179, 433

Burrows, D. N., Romano, P., Falcone, A., et al. 2005, Science, 309, 1833

Cannizzo, J. K., Gehrels, N., \& Vishniac, E. T. 2004, ApJ, 601, 380

Daigne, F., \& Mochkovitch, R. 1998, MNRAS, 296, 275

Drenkhahn, G. 2002, A\&A, 387, 714

Drenkhahn, G., \& Spruit, H. C. 2002, A\&A, 391, 1141

Fan, Y.-Z., Dai, Z.-G., Huang, Y.-F., \& Lu, T. 2002, Ch. J. Astron. Astrophys., 2, 449

Fan, Y. Z., Wei, D. M., \& Wang, C. F. 2004, A\&A, 424, 477

Fox, D. W., Price, P. A., Soderberg, A. M., et al. 2003, ApJ, 586, L5

Genet, F., Daigne, F., \& Mochkovitch, R. 2006, A\&A, 457, 737

Genet, F., Daigne, F., \& Mochkovitch, R. 2007, MNRAS, 381, 732

Giannios, D. 2006a, A\&A, 455, L5

Giannios, D. 2006b, A\&A, 457, 763

Giannios, D., \& Spruit, H. C. 2006, A\&A, 450, 887

Giannios, D., \& Spruit, H. C. 2007, A\&A, 469, 1

Goodman, J. 1986, ApJ, 308, L47

Granot, J., \& Taylor, G. B. 2005, ApJ, 625, 263

Granot, J., Miller, M., Piran, T., Suen, W. M., \& Hughes, P. A. 2001, Gamma-ray Bursts in the Afterglow Era, 312

Koers, H. B. J., \& Giannios, D. 2007, A\&A, 471, 395

Kumar, P., \& Panaitescu, A. 2003, MNRAS, 346, 905

Lazzati, D., Covino, S., Gorosabel, J., et al. 2004, A\&A, 422, 121

Levinson, A., \& Eichler, D. 1993, ApJ, 418, 386

Li, Z.-Y., Chiueh, T., \& Begelman, M. C. 1992, ApJ, 394, 459

Lyutikov, M. 2005, [arXiv: astro-ph/0503505]

Lyutikov, M. 2006, New J. Phys., 8, 119

Lyutikov, M., \& Blandford, R. 2003, [arXiv: astro-ph/0312347]

McMahon, E., Kumar, P., \& Piran, T. 2006, MNRAS, 366, 575

Medvedev, M. V., \& Loeb, A. 1999, ApJ, 526, 697

Meliani, Z., Keppens, R., Casse, F., \& Giannios, D. 2007, MNRAS, 376, 1189

Mészáros, P., \& Rees, M. J. 1997a, ApJ, 476, 232

Mészáros, P., \& Rees, M. J. 1997b, ApJ, 482, L29

Michel, F. C. 1969, ApJ, 158, 727

Mimica, P., Aloy, M.A., Müller, E., \& Brinkmann, W., 2005, A\&A, 441, 103

Mimica, P., Aloy, M. A., \& Müller, E., 2007a, A\&A, 466, 93

Mimica, P., Giannios, D., \& Aloy, M. A., 2007b, in preparation

Mundell, C. G., Steele, I. A., Smith, R. J., et al. 2007a, Science, 315, 1822

Mundell, C. G., Melandri, A., Guidorzi, C., et al. 2007b, ApJ, 660, 489

Nakar, E., \& Piran, T. 2002, ApJ, 572, L139

Paczynski, B. 1986, ApJ, 308, L43

Paczyński, B., \& Xu, G. 1994, ApJ, 427, 708

Rees, M. J., \& Mészáros, P. 1994, ApJ, 430, L93

Rezzolla, L., \& Zanotti, O. 2001, J. Fluid Mech., 449, 395

Ruffert, M., \& Janka, H.-T. 1998, A\&A, 338, 535

Sari, R., \& Piran, T. 1995, ApJ, 455, L143

Sari, R., \& Piran, T. 1999, ApJ, 520, 641

Spitkovsky, A. 2007, ApJ, submitted, [arXiv:0706.3126]

Spruit, H. C., Daigne, F., \& Drenkhahn, G. 2001, A\&A, 369, 694

Thompson, C. 1994, MNRAS, 270, 480

Thompson, C. 2006, ApJ, 651, 333

Uhm, Z. L., \& Beloborodov, A. M. 2007, ApJ, 665, L93

Usov, V. V. 1992, Nature, 357, 472

Uzdensky, D. A., \& MacFadyen, A. I. 2006, ApJ, 647, 1192

Van Putten, M. H. P. M., \& Ostriker, E. C. 2001, ApJ, 552, L31

Vlahakis, N., \& Königl, A. 2003, ApJ, 596, 1080

Woosley, S. E. 1993, ApJ, 405, 273

Zhang, B., \& Kobayashi, S. 2005, ApJ, 628, 315 (ZK05)

Zhang, B., Kobayashi, S., \& Mészáros, P. 2003, ApJ, 595, 950

Zhang, B., Fan, Y. Z., Dyks, J., et al. 2006, ApJ, 642, 354 